\title{
Blunted Tungsten Tip Cleaning by Nitrogen Gas Etching at Room Temperature without Tip Heating and Cooling.
}

\author{
In-Yong Park, Takashi Ogawa, Boklae Cho, Cheolsu Han, Ju Hwang Kim, Sang Jung Ahn* \\ Center for Advanced Instrumentation, Division of Industrial Metrology, Korea Research Institute of \\ Standards and Science (KRISS), 267 Gajeong-Ro, Yuseong-Gu, Daejeon 305-340, South Korea
}

For a long time, the ultra-sharp tips are used for high resolution microscope, such as scanning tunneling microscope (STM), atomic force microscope (ATM), field emission scanning electron microscope (FE-SEM). In addition, helium ion microscope (HIM), which has sub-nanometer imaging resolution, employs the tip as gas field ionization source (GFIS) in recent years. There are a lot of required vital conditions of tip fabrication and source characterization for the high performance of microscopes. Among them, tip cleanliness and operation temperature are very essential for the stable and high charged particle beam current. The contaminants on the surface of tip will undoubtedly cause instabilities in the tunnel junction between tip and gases and abruptly increases fluctuation of the charged particle beam current, thus affecting the image quality and noise level. Also, its requirements for liquid nitrogen cooling make the microscope system complicated, expensive maintenance cost and price of apparatus. This study presents a simple and efficient way to clean the blunted tungsten tip using a field-assisted nitrogen etching at room temperature without tip heating and cooling methods.

Tungsten is commonly adopted for tip material because extremely sharp tip can be easily fabricated through electrochemical etching and it has higher evaporation field than ionization filed of rare gases. However, it is well known fact that oxide layers are created on the tungsten tip surface during the electrochemical etching, being exposed to the atmospheric conditions. In addition, residual gases of chamber and impurity of imaging gases are easily absorbed on tip surface. In order to eliminate the contaminants, there are representative two methods. The one is annealing and the other is field evaporation (desorption). In case of annealing, the contaminant layer is removed at approximately when tips are heated at $\sim 1000 \mathrm{~K}$ for several seconds or minutes. However, high temperature could induce the surface diffusion which causes atoms to migrate from the tip apex to tip shank, thereby increasing the radius of tip after annealing [1]. Besides, the optimal heating conditions might be changed from tip to tip since these resistance and length are different. On the other hand, field evaporation can get rid of the contaminants intensively in the vicinity of the tip apex. Unfortunately, many researchers say that the longer duration of tip usage, the duller tip will become. This causes the increase in applying voltage for field evaporation and eventually replacing it with a new tip.

The nitrogen gas etching with tungsten surface can sharpen the tip until tip apex is terminated atomically defined level [2], this can be explained by nitrogen gas adsorption and tungsten atom evaporation. When the nitrogen gases adsorb onto the tungsten surface, it penetrates under several surface layers and make the tungsten atoms protruded. These protrusions are easily evaporated by field enhancement. The successive field evaporations also make the blunted tip resharpened with removal of peripheral tungsten atoms, so we applied this phenomenon for cleaning the blunted tip in which field evaporation rarely occur with maximum $10 \mathrm{kV}$, which is our experimental condition, due to large radius of tip. Firstly, a tungsten tip made by an electrochemical etching in $\mathrm{KOH}$ solution is transferred to the ultra high vacuum (UHV) chamber with a background pressure of $\sim 10^{-8} \mathrm{~Pa}$. We can easily observe field ion microscope (FIM) images of the clean tungsten crystalline surface plane just with field evaporation, where tip voltage is around $5 \mathrm{kV}$ and helium gas pressure is $\sim 10^{-4} \mathrm{~Pa}$. The tip is gradually blunted after FIM experiments and exposure to atmosphere in many times. Eventually, because tip voltage can just reaches 
to maximum $10 \mathrm{kV}$ in our experiment system, the dull tip cannot be cleaned only with field evaporation since the induced field at the end of tip is not enough to evaporate the contaminants. At this point, we additionally filled the chamber with nitrogen gas of partial pressure around $10^{-6} \sim 10^{-5} \mathrm{~Pa}$ and applied the extraction voltage up to $10 \mathrm{kV}$, not having annealing step which could easily blunt tip. As shown in figure 1, subsequent field evaporations of tip periphery cause electric field of the tip apex to increase. At last, the clean crystalline surface plane appears with decreasing voltage slowly down to $\sim 6 \mathrm{kV}$. Sequentially, controlling nitrogen partial pressure and extraction voltage carefully and appropriately, we can make the single atom tip reproducibly even at room temperature as figure 2.

In conclusions, dull tip which is blunted by usage or originally can be cleaned only with field-assited nitrogen etching. Since we do not use the tip heating for cleaning, it is anticipated that the technique considered here can be readily reduce the possibility to get dulled by such annealing step. Furthermore, frequent tip changing could be avoided because dull tip can obtain a new life by in situ regeneration in UHV. Thus, the tip life and operation time can be increased, including simple microscopy design and operation in room temperature.

\section{References}

[1] Lucier, A. S., Center for the Physics of Materials McGill University, thesis (2005)

[2] Rezeq, M., Pitters, J. and Wolkow, R., J Chem Phys. 124 (2006), 204716

[3] This research was supported by Nano Material Technology Development Program through the National Research Foundation of Korea (NRF) funded by the Ministry of Education, Science and Technology (grant number: 2011-0030233).

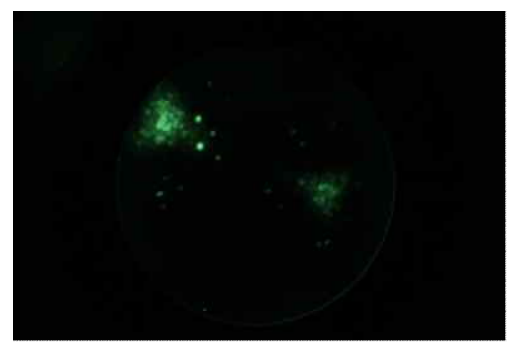

(1)

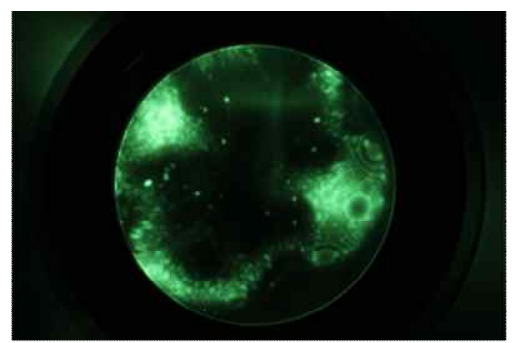

(2)

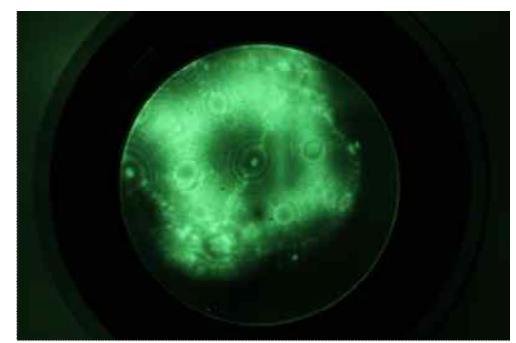

(3)

Figure 1. FIM images during field-assisted nitrogen gas cleaning of blunted tip in real time. Order of numbers means flow of time.

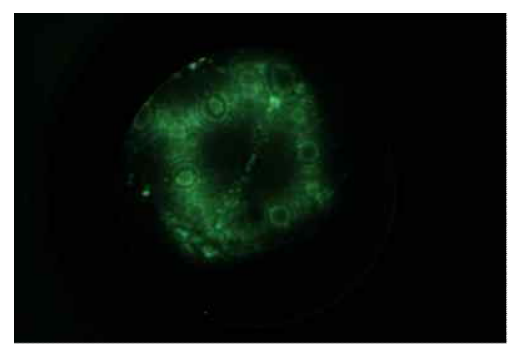

(1)

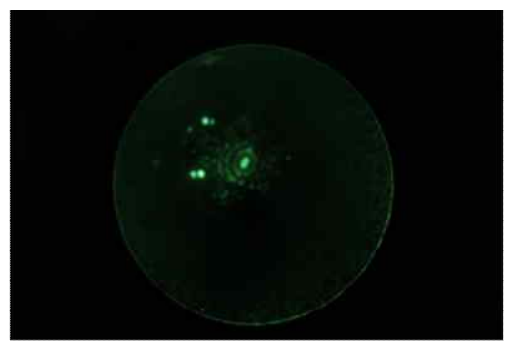

(2)

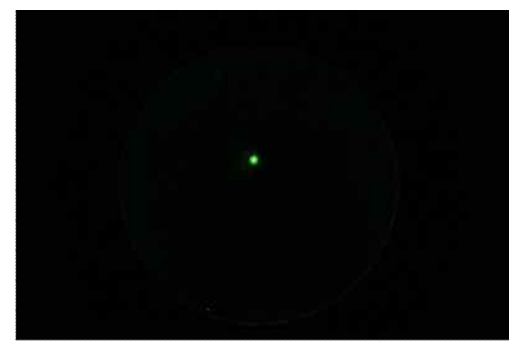

(3)

Figure 2. FIM images during fabrication of single atom tip with field-assisted nitrogen gas etching in real time. Order of numbers means flow of time.

*Corresponding author: sjahn@kriss.re.kr 